\title{
Analytical solution for a viscoelastic plate on a Pasternak foundation
}

\section{Khazanovich, Lev; Levenberg, Eyal}

\section{Published in:}

Road Materials and Pavement Design

Link to article, DOI:

10.1080/14680629.2018.1530693

Publication date:

2020

Document Version

Peer reviewed version

Link back to DTU Orbit

Citation (APA):

Khazanovich, L., \& Levenberg, E. (2020). Analytical solution for a viscoelastic plate on a Pasternak foundation. Road Materials and Pavement Design, 21(3), 800-820. https://doi.org/10.1080/14680629.2018.1530693

\section{General rights}

Copyright and moral rights for the publications made accessible in the public portal are retained by the authors and/or other copyright owners and it is a condition of accessing publications that users recognise and abide by the legal requirements associated with these rights.

- Users may download and print one copy of any publication from the public portal for the purpose of private study or research.

- You may not further distribute the material or use it for any profit-making activity or commercial gain

- You may freely distribute the URL identifying the publication in the public portal

If you believe that this document breaches copyright please contact us providing details, and we will remove access to the work immediately and investigate your claim 


\section{Analytical Solution for a Viscoelastic Plate on a Pasternak Foundation}

Lev Khazanovich ${ }^{\mathrm{a}}$ and Eyal Levenberg ${ }^{\mathrm{b} *}$

${ }^{a}$ Civil and Environmental Engineering, University of Pittsburgh, Pittsburgh 15261, Pennsylvania

${ }^{b}$ Civil Engineering, Technical University of Denmark, Kongens Lyngby 2800, Denmark

* Corresponding author. Email: eylev@byg.dtu.dk

This work contributed an analytical solution to the problem of an infinite viscoelastic plate supported on a Pasternak foundation and subjected to axisymmetric normal loading. The derivation was based on defining a set of iterative functions, each containing information on the plate's relaxation modulus and on the time-variation of the loading. By writing the sought solution as a linear combination of these functions it was shown how to decompose the original viscoelastic problem into a set of independent elastic plate problems for which analytical solutions exist. Thus, the plate's quasistatic deflection evolution at any point of interest was provided in closed-form, without resorting to integral transform techniques. The formulation was applied and subsequently validated for several test cases, demonstrating that a very small set of elastic solutions is needed for generating a highly accurate viscoelastic result. Overall, the proposed solution is deemed well suited for engineering calculations, as a computational kernel for backcalculation, and for benchmarking numerical solutions.

Keywords: plate; Pasternak foundation; viscoelasticity; integral operator; analytical solution

\section{Introduction}

The problem of a loaded elastic plate resting on a continuous support is of interest in diverse study fields. Example applications include: (i) mechanics of concrete pavements (Westergaard, 1948; Pronk, 1993; Khazanovich and Ioannides, 1993; Van Cauwelaert, Stet, \& Jasienski, 2002), (ii) mechanics of raft foundations (Selvadurai, 1979; Hemsley, 2000), (iii) mechanics of floating ice sheets (Wyman, 1950; Kerr \& Palmer, 1972), (iv) plate tectonics (Brotchie \& Silvester, 1969; Watts, 2001), and (v) foam mechanics (Altenbach \& Eremeyev, 2009). A closely related problem, dealing with a viscoelastic plate resting on a continuous support, is of particular relevance to the pavement arena whenever structures include one or more layers that exhibit time-dependent properties. Early investigations in 
this direction were focused on asphalt pavements (Pister \& Monismith, 1960; Pister \& Westmann, 1962; Radovskii, 1979). More recently, a simplified form of the problem, involving an infinite viscoelastic beam on a spring support was utilized for quantifying energy loses during wheel rolling (Louhghalam, Akbarian, \& Ulm, 2013). Other unexplored applications for which such modeling is well suited include pavements surfaced with semi-rigid materials such as grouted Macadam (Anderton, 2000; Pożarycki et al., 2018) or rubberized Portland cement concrete (Shu \& Huang, 2014).

Exiting studies dealing with viscoelastic plates (e.g., Mase, 1961; Pister, 1961; Robertson, 1971; Marvin, 1972; Hewitt \& Mazumdar, 1974; Takagi, 1978; Radovskii, 1979; Misra \& Samanta, 1980; Hui, 1986; Elattary, Cozzarelli, \& Shaw, 1988; Hosking, Sneyd, \& Waugh, 1988; Altenbach \& Eremeyev, 2009) have largely employed the elastic-viscoelastic correspondence principle. This type of approach mandates Laplace transform inversion, and can therefore yield closed-form solutions only when analytical transformations exist. This essentially means restricting the plate's viscoelastic properties to simple Kelvin-Voigt or Maxwell models. For considering more realistic situations, involving broadband viscoelastic properties expressed through empirical formulas (Smith, 1971) or through multi-term Prony series (Williams, 1964), it is unavoidable to resort to numerical transform inversion techniques.

This study is motivated by the many advantages analytical solutions offer over numerical schemes (Selvadurai, 2007), especially in terms of accuracy, precision, computational stability, and in many cases computational speed. These reasons render analytic solvers the preferred tool for engineering first-order calculations, for benchmarking numerical solutions, and as a computational kernel when carrying out inverse analysis. Subsequently, the purpose here is to contribute an analytical solution for the problem of an infinite plate supported on a Pasternak foundation. The plate is composed of a linear viscoelastic material modelled with realistic broadband properties, and the loading is spread uniformly over a circular area with time-varying stress intensity. The solution aims to deliver the plate's quasistatic deflection evolution at any point of interest during a desired time interval without resorting to integral transform techniques of any kind. A Pasternak support was selected because it is considered a universal two-parameter foundation (Kerr, 1964), mathematically generalizing similar models that either introduce some interaction between the Winkler springs 
(Filonenko-Borodich, 1940; Hetenyi, 1946) or attempt to simplify a continuum (Reissner, 1958; Vlasov \& Leont'ev, 1966).

Presented first is a closed-form solution for an elastic plate. A complete step-by-step derivation of this solution, following Korenev (1954), in included in Appendix A. Based on the elastic solution, a step-by-step derivation of a viscoelastic plate solution is presented next. This is followed by specific examples to demonstrate the formulation and to further clarify the underlying computations. Lastly, a validation scheme for the solution correctness is offered, applied, and discussed. The paper terminates with a short summery and some remarks.

\section{The elastic problem}

Consider a linear elastic plate of constant thickness and infinite dimensions loaded by an axisymmetric stress distribution of uniform intensity (Figure 1). The plate is supported on a Pasternak foundation composed of a bed of closely spaced identical linear springs (i.e. a Winkler foundation) mechanically coupled by a layer attached to their top that can only offer resistance to shear deformations. A cylindrical coordinate system is positioned at the midsurface of the undeformed plate. The radial $r$-axis points in any direction that is parallel to the plate's undeformed surface boundaries. The vertical $z$-axis coincides with the axis of rotational symmetry and points downward towards the support.

The differential equation the governs the plate's vertical displacements (i.e., deflections) under the above-described loading conditions is

$$
D \nabla^{4} w_{a}(r)-G \nabla^{2} w_{a}(r)+k w_{a}(r)=q H(a-r)
$$

where $D=12^{-1} E h^{3}\left(1-v^{2}\right)^{-1}$ is the plate's flexural rigidity (units of force $\times$ length), in which $E$ is Young's modulus, $h$ is the thickness, and $v$ is Poisson's ratio. The deflection at a radial coordinate $r$ is denoted by $w_{a}(r)$ and $\nabla^{4}$ is the twice-applied two-dimensional axisymmetric Laplacian operator $\nabla^{2}=d^{2} / d r^{2}+r^{-1} d / d r$. The Winkler foundation modulus is denoted by $k$ (units of force/length ${ }^{3}$ ), while $G$ represents the intensity of shear interaction between the spring elements (units of force/length). The applied stress acts in the $z$-axis direction with uniform intensity $q$ over a circular area with radius $a$. This stress distribution is mathematically expressed as a function of the radial coordinate by means of the Heaviside step function $H(\cdot)$. 


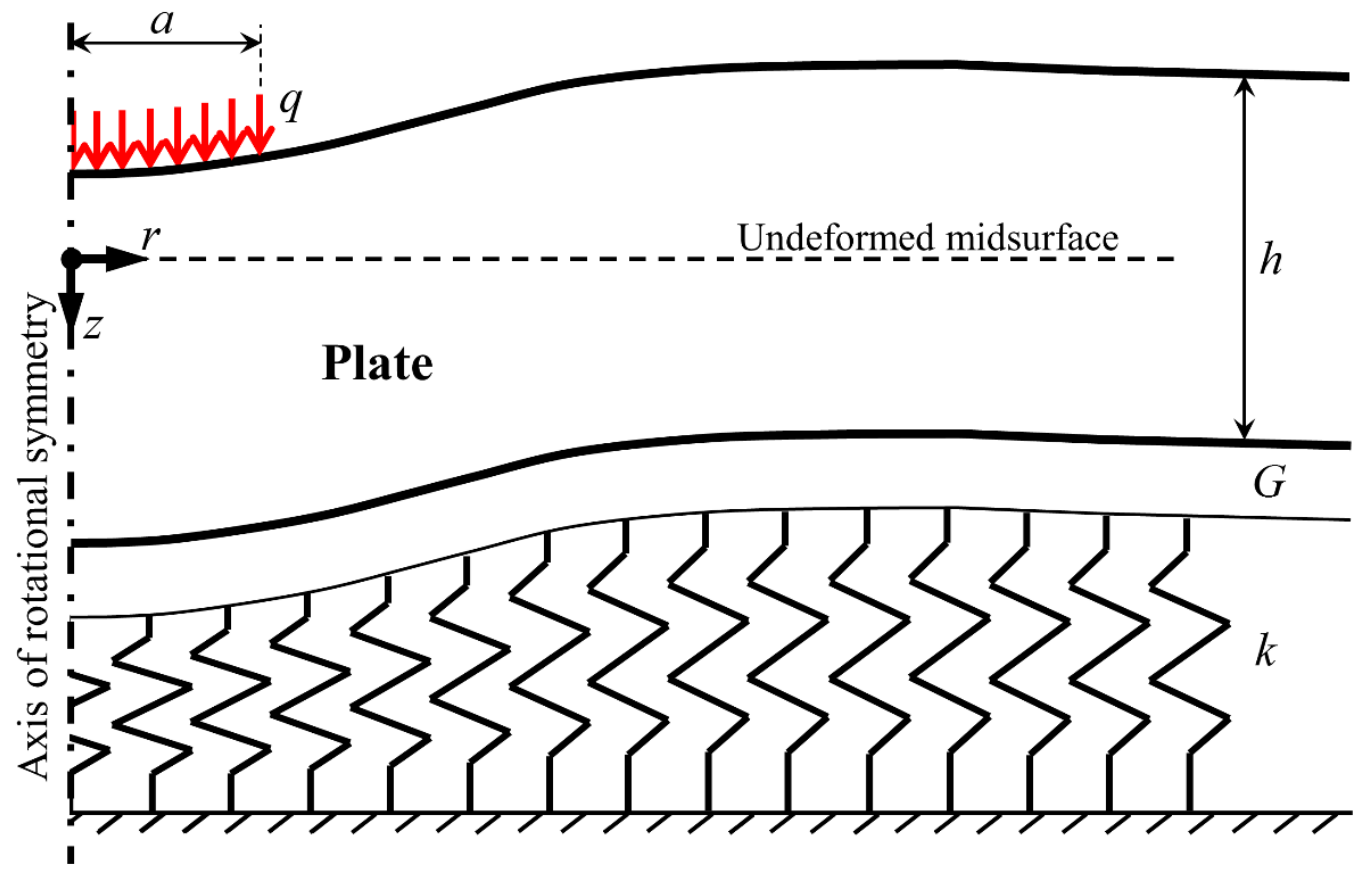

Figure 1. Plate on a Pasternak foundation loaded axisymmetrically.

Following Korenev (1954), an analytical solution for $w_{a}(r)$ is given by two separate closedform expressions

$$
w_{a}(r)=\left\{\begin{array}{l}
r \leq a, \frac{\pi \alpha q}{4 k \sin 2 \phi}\left(\begin{array}{l}
\frac{2}{\pi \alpha}\left(i e^{-2 i \phi}-i e^{2 i \phi}\right)+e^{-i \phi} H_{1}^{(1)}\left(\alpha e^{i \phi}\right) J_{0}\left(s e^{i \phi}\right) \\
+e^{i \phi} H_{1}^{(2)}\left(\alpha e^{-i \phi}\right) J_{0}\left(s e^{-i \phi}\right)
\end{array}\right) \\
r>a, \quad \frac{\pi \alpha q}{4 k \sin 2 \phi}\left(e^{-i \phi} H_{0}^{(1)}\left(s e^{i \phi}\right) J_{1}\left(\alpha e^{i \phi}\right)+e^{i \phi} H_{0}^{(2)}\left(s e^{-i \phi}\right) J_{1}\left(\alpha e^{-i \phi}\right)\right)
\end{array}\right.
$$

where $\alpha=a / l$ is a non-dimensional loaded radius, $s=r / l$ is a non-dimensional radial coordinate, and $l=\sqrt[4]{D / k}$ is the characteristic length or the so-called radius of relative stiffness. Moreover, $J_{0}$ and $J_{1}$ are Bessel functions of the first kind of order zero and one (respectively), $H_{0}^{(1)}$ and $H_{1}^{(1)}$ are Hankel functions of the first kind of order zero and one (respectively), $H_{0}^{(2)}$ and $H_{1}^{(2)}$ are Hankel functions of the second kind of order zero and one (respectively), $i=\sqrt{-1}$, and $\phi$ is given by the expression

$$
\phi=\frac{1}{2}\left(\pi+\arctan \left(\frac{\sqrt{1-b^{2}}}{-b}\right)\right)
$$


wherein $b=G\left(2 k l^{2}\right)^{-1}$ is a positive dimensionless parameter presumed smaller than unity, i.e., $0 \leq b \leq 1$. This supposition is equivalent to $k \geq 0.25 G^{2} / D$; it essentially means that vertical stiffness, and not shear interaction effects, dominate the mechanical response of the plate-foundation system (Kerr \& El-Sibaie, 1989). This appears to be the case for soil-structure interaction problems (Kerr, 1965; Fwa, Shi \& Tan, 1996). Accordingly, it can be deduced that $\pi / 4 \leq \phi \leq \pi / 2$ with the special case of $\phi=\pi / 4$ (i.e., $b \rightarrow 0$ ) representing a Winkler foundation. Appendix $\mathrm{A}$ is dedicated to the rederivation of Equation (2).

\section{The viscoelastic problem}

Consider a plate composed of a linear isotropic viscoelastic material characterized by a relaxation function $E(t)$ and a constant (time-independent) Poisson's ratio $v_{0}$. The corresponding onedimensional constitutive equation of the relaxation type may be written as (Flügge, 1975)

$$
\sigma(t)=E_{0} \varepsilon(t)+\int_{0^{+}}^{t} \frac{d E(t-\tau)}{d(t-\tau)} \varepsilon(\tau) d \tau
$$

where $\sigma(t)$ is the stress history and $\varepsilon(t)$ is the strain history - both assumed zero for $t<0, E_{0}$ is the instantaneous modulus, i.e., $E_{0}=\lim _{t \rightarrow 0} E(t)$, and $\tau$ is a time-like integration variable. By defining the memory kernel $R(t)$ (units reciprocal to time)

$$
R(t)=\frac{1}{E_{0}} \frac{d E(t)}{d t}
$$

The constitutive relation in Equation (4) can be written alternatively as

$$
\sigma(t)=E_{0}(1+\tilde{R}) \varepsilon(t)
$$

with $\tilde{R}$ as an integral operator that maps $x \mapsto \tilde{R} x$ where $x(t)$ is some function of time

$$
\tilde{R} x(t)=\int_{0^{+}}^{t} R(t-\tau) x(\tau) d \tau
$$

Note that $\tilde{R}$ is a linear operator, and that units are unaffected by the mapping, i.e., $\tilde{R} x$ retains the units of $x$. 
Given the above definitions, and with reference to Equation (1), the governing equation for a viscoelastic plate resting on a Pasternak foundation (and loaded over a circle) becomes integrodifferential

$$
D_{0}(1+\tilde{R}) \nabla^{4} w_{a}^{v e}(r, t)-G \nabla^{2} w_{a}^{v e}(r, t)+k w_{a}^{v e}(r, t)=q_{0} H(a-r) f(t)
$$

in which $w_{a}^{v e}(r, t)$ is the sought plate deflection at any radial coordinate and time of interest, $D_{0}=12^{-1} E_{0} h^{3}\left(1-v_{0}^{2}\right)^{-1}$ is the instantaneous flexural rigidity of the plate, $q_{0}$ is a constant with stress units, and $f(t)$ is a non-dimensional function that equals zero for negative time and represents the variation of the applied stress intensity for $t \geq 0$.

\subsection{Proposed method}

Utilizing the integral operator $\tilde{R}$ defined in Equation (7), combined with the stress variation function $f(t)$ introduced in Equation (8), a set of $N$ dimensionless time functions is iteratively constructed as follows (Volterra, 1959; Zevin, 1971)

$$
\begin{aligned}
f_{1}(t)= & f(t) \\
f_{2}(t)= & \tilde{R} f_{1}(t)=\tilde{R} f(t) \\
f_{3}(t)= & \tilde{R} f_{2}(t)=\tilde{R}^{2} f(t) \\
& \vdots \\
f_{N}(t)= & \tilde{R} f_{N-1}(t)=\tilde{R}^{N-1} f(t)
\end{aligned}
$$

This set of iterative functions is then linearly combined to express the sought plate deflection

$$
w_{a}^{v e}(r, t)=\sum_{n=1}^{N} w_{n} f_{n}(t)
$$

where the $w_{n}$ 's (units of length) are each a function of the radial coordinate only, i.e., $w_{n}=w_{n}(r)$. Mathematically, Equation (10) is only exact at the limit, as $N \rightarrow \infty$. Practically however, only a small set of iterative functions is necessary for attaining high accuracy. This is due to fading memory considerations, which mandates $E(t)$ to be a monotonically decreasing function of time.

Insertion of Equation (10) into Equation (8) gives

$$
D_{0}\left(\sum_{n=1}^{N} \nabla^{4} w_{n}\left(f_{n}(t)+\tilde{R} f_{n}(t)\right)\right)-G\left(\sum_{n=1}^{N} \nabla^{2} w_{n} f_{n}(t)\right)+k\left(\sum_{n=1}^{N} w_{n} f_{n}(t)\right)=q_{0} H(\mathrm{a}-r) f(t)
$$


and by virtue of Equation (9) the leftmost bracketed summation term in Equation (11) becomes

$$
\sum_{n=1}^{N} \nabla^{4} w_{n}\left(f_{n}(t)+f_{n+1}(t)\right)=\nabla^{4} w_{1} f_{1}(t)+\sum_{n=2}^{N}\left(\nabla^{4} w_{n-1}+\nabla^{4} w_{n}\right) f_{n}(t)+\nabla^{4} w_{N} f_{N+1}(t)
$$

At this point, following Zevin (1971), it is proposed to represent $f_{N+1}(t)$ by a linear combination of its predecessors

$$
f_{N+1}(t) \approx \sum_{n=1}^{N} c_{n} f_{n}(t)
$$

wherein the $c_{n}$ 's are real unitless constants and $c_{1}=0$. For a finite time interval over which the solution is sought, i.e., $0 \leq t \leq T$, the correctness of this representation is governed by the choice of $N$. The numerical values of the $c_{n}$ 's $(n=2 \ldots N)$ may be found by minimizing the error term Err defined as follows

$$
E r r=\int_{0}^{T}\left(\operatorname{err}_{N}(t)\right)^{2} d t=\int_{0}^{T}\left(\sum_{n=2}^{N} c_{n} f_{n}(t)-f_{N+1}(t)\right)^{2} d t
$$

where the function $\operatorname{err}_{N}(t)$ embodies the error variation within the analysis time interval $0 \leq t \leq T$ that is associated with the representation of $f_{N+1}(t)$ by a linear combination of the iterative functions $f_{2}(t), \ldots, f_{N}(t)$. Equation (14) is essentially a continuous least squares formulation, solved from the following set of $N-1$ equations

$$
\begin{gathered}
d E r r / d c_{2}=0 \\
d E r r / d c_{3}=0 \\
\vdots \\
d E r r / d c_{N}=0
\end{gathered}
$$

Consequently, by means of Equation (13), Equation (12) becomes

$$
\begin{aligned}
\nabla^{4} w_{1} f_{1}(t)+\sum_{n=2}^{N} & \left(\nabla^{4} w_{n-1}+\nabla^{4} w_{n}\right) f_{n}(t)+\nabla^{4} w_{N} \sum_{n=1}^{N} c_{n} f_{n}(t)= \\
= & \nabla^{4} w_{1} f_{1}(t)+\sum_{n=2}^{N}\left(\nabla^{4} w_{n-1}+\nabla^{4} w_{n}+c_{n} \nabla^{4} w_{N}\right) f_{n}(t)
\end{aligned}
$$

From here onward, it is instructive to employ matrix notation, and define the following $N \times 1$ column matrices 


$$
\begin{aligned}
& \mathbf{f}=\left\{f_{1}(t), f_{2}(t), \ldots, f_{N-1}(t), f_{N}(t)\right\}^{\mathrm{T}} \\
& \mathbf{w}=\left\{w_{1}, w_{2}, \ldots, w_{N-1}, w_{N}\right\}^{\mathrm{T}} \\
& \mathbf{w}_{\mathbf{2}}=\left\{\nabla^{2} w_{1}, \nabla^{2} w_{2}, \ldots, \nabla^{2} w_{N-1}, \nabla^{2} w_{N}\right\}^{\mathrm{T}} \\
& \mathbf{w}_{\mathbf{4}}=\left\{\nabla^{4} w_{1}, \nabla^{4} w_{2}, \ldots, \nabla^{4} w_{N-1}, \nabla^{4} w_{N}\right\}^{\mathrm{T}} \\
& \mathbf{q}=\left\{q_{0} H(a-r), 0, \ldots, 0,0\right\}^{T}
\end{aligned}
$$

as well as the following $N \times N$ square matrix (wherein zero elements have been omitted for clarity)

$$
\mathbf{M}=\left[\begin{array}{cccccc}
1 & & & & & \\
1 & 1 & & & & c_{2} \\
& 1 & 1 & & & c_{3} \\
& & \ddots & \ddots & & \vdots \\
& & & 1 & 1 & c_{N-1} \\
& & & & 1 & 1+c_{N}
\end{array}\right]
$$

from which Equation (10) becomes $w_{a}^{v e}(r, t)=\mathbf{w}^{\mathrm{T}} \mathbf{f}$, and Equation (11) becomes

$$
D_{0}\left(\mathbf{M} \mathbf{w}_{4}\right)^{\mathrm{T}} \mathbf{f}-G \mathbf{w}_{2}^{\mathrm{T}} \mathbf{f}+k \mathbf{w}^{\mathrm{T}} \mathbf{f}=\mathbf{q}^{\mathrm{T}} \mathbf{f}
$$

This expression can be simplified by eliminating $\mathbf{f}$ appearing in all terms, and by removing the transpose operation, to yield a set of $N$ coupled equations

$$
D_{0} \mathbf{M} \mathbf{w}_{4}-G \mathbf{w}_{2}+k \mathbf{w}=\mathbf{q}
$$

As means of eliminating the interaction between the equations, a Jordan (eigen) decomposition is performed on $\mathbf{M}$

$$
\mathbf{M}=\mathbf{V A} \mathbf{V}^{-1}
$$

where $\mathbf{A}$ is a diagonal matrix composed of $N$ eigenvalues $\lambda_{n}$ 's of $\mathbf{M}$

$$
\mathbf{A}=\left[\begin{array}{llll}
\lambda_{1} & & & \\
& \lambda_{2} & & \\
& & \ldots & \\
& & & \lambda_{N}
\end{array}\right]
$$


while $\mathbf{V}$ is a $N \times N$ square matrix whose columns are the eigenvectors of $\mathbf{M}$. Insertion of Equation (25) into Equation (24), and then left-multiplying each of the terms by $\mathbf{V}^{-1}$ results in

$$
D_{0} \mathbf{A} \mathbf{V}^{-1} \mathbf{w}_{\mathbf{4}}-G \mathbf{V}^{-1} \mathbf{w}_{\mathbf{2}}+k \mathbf{V}^{-1} \mathbf{w}=\mathbf{V}^{-1} \mathbf{q}
$$

Introducing the following new $N \times 1$ column matrices

$$
\begin{aligned}
& \mathbf{w}^{*}=\mathbf{V}^{-1} \mathbf{w}=\left\{w_{1}^{*}, w_{2}^{*}, \ldots, w_{N-1}^{*}, w_{N}^{*}\right\}^{\mathrm{T}} \\
& \mathbf{w}_{\mathbf{2}}^{*}=\mathbf{V}^{-1} \mathbf{w}_{\mathbf{2}}=\left\{\nabla^{2} w_{1}^{*}, \nabla^{2} w_{2}^{*}, \ldots, \nabla^{2} w_{N-1}^{*}, \nabla^{2} w_{N}^{*}\right\}^{\mathrm{T}} \\
& \mathbf{w}_{\mathbf{4}}^{*}=\mathbf{V}^{-1} \mathbf{w}_{\mathbf{4}}=\left\{\nabla^{4} w_{1}^{*}, \nabla^{4} w_{2}^{*}, \ldots, \nabla^{4} w_{N-1}^{*}, \nabla^{4} w_{N}^{*}\right\}^{\mathrm{T}}
\end{aligned}
$$

a decoupled set of $N$ equations is obtained

$$
D_{0} \mathbf{A} \mathbf{w}_{\mathbf{4}}^{*}-G \mathbf{w}_{\mathbf{2}}^{*}+k \mathbf{w}^{*}=\mathbf{q}^{*}
$$

wherein $\mathbf{q}^{*}=\mathbf{V}^{-1} \mathbf{q}$.

\subsection{Solution for a viscoelastic plate}

Essentially, each line in Equation (31) resembles the elastic formulation in Equation (1). Therefore, the elastic solution in Equation (2) directly gives $w_{1}^{*}, \ldots, w_{N}^{*}$ in any desired order. It is noted that the 'flexural rigidity' at the $n$th equation, which equals the product $D_{0} \lambda_{n}$, can be complex. This is because some of the eigenvalues in A may be complex. Hence, derived entities such as $l, b$, and $\phi$ can also become complex. Similarly, the 'stress intensities' $\mathbf{q}^{*}$ may contain complex elements. Nonetheless, despite the fact that intermediate calculation steps yield complex results, the final (sought) viscoelastic solution is real, calculated from

$$
w_{a}^{v e}(r, t)=\left(\mathbf{V} \mathbf{w}^{*}\right)^{\mathrm{T}} \mathbf{f}
$$

or alternatively, based on Equation (2), an analytic closed-form expression is attained for a viscoelastic plate on a Pasternak foundation 


$$
w_{a}^{v e}(r, t)=\left\{\begin{array}{l}
r \leq a, \quad \sum_{n=1}^{N} \frac{\pi \alpha_{n} q_{n}^{*} f_{n}(t)}{4 k \sin 2 \phi_{n}}\left(\begin{array}{l}
\frac{2}{\pi \alpha_{n}}\left(i e^{-2 i \phi_{n}}-i e^{2 i \phi_{n}}\right)+e^{-i \phi_{n}} H_{1}^{(1)}\left(\alpha_{n} e^{i \phi_{n}}\right) J_{0}\left(s_{n} e^{i \phi_{n}}\right) \\
+e^{i \phi_{n}} H_{1}^{(2)}\left(\alpha_{n} e^{-i \phi_{n}}\right) J_{0}\left(s_{n} e^{-i \phi_{n}}\right)
\end{array}\right) \\
r>a, \sum_{n=1}^{N} \frac{\pi \alpha_{n} q_{n}^{*} f_{n}(t)}{4 k \sin 2 \phi_{n}}\left(\begin{array}{l}
e^{-i \phi_{n}} H_{0}^{(1)}\left(s_{n} e^{i \phi_{n}}\right) J_{1}\left(\alpha_{n} e^{i \phi_{n}}\right) \\
+e^{i \phi_{n}} H_{0}^{(2)}\left(s_{n} e^{-i \phi_{n}}\right) J_{1}\left(\alpha_{n} e^{-i \phi_{n}}\right)
\end{array}\right)
\end{array}\right)
$$

wherein $l_{n}=\sqrt[4]{D_{0} \lambda_{n} / k}$, from which $b_{n}=G\left(2 k l_{n}^{2}\right)^{-1}, \phi_{n}=0.5 \pi+0.5 \arctan \left(-\sqrt{b_{n}^{-2}-1}\right), \alpha_{n}=a / l_{n}$, $s_{n}=r / l_{n}$, and $q_{n}^{*}$ is the $n$th element in $\mathbf{q}^{*}$ excluding the Heaviside term $H(a-r)$ in Equation (21).

\section{Illustration of solution method}

As means of illustrating the solution method, a plate with thickness $h=200 \mathrm{~mm}$ is considered, composed of a linear viscoelastic solid material characterized by a constant Poisson's ratio $v_{0}=0.20$ and a relaxation function of the form $E(t)=E_{\infty}+\left(E_{0}-E_{\infty}\right) e^{-t / \rho}$ wherein $E_{\infty}=10^{4} \mathrm{MPa}$ is the equilibrium modulus, $E_{0}=4 \cdot 10^{4} \mathrm{MPa}$ is the instantaneous modulus, and $\rho=2 \mathrm{~s}$ is the relaxation time. Subsequently, $D_{0}=2.778 \cdot 10^{10} \mathrm{Nmm}$, and the memory kernel, Equation (5), is $R(t)=-0.375 e^{-t / 2}$. The time functions $E(t)$ and $R(t)$ are displayed in Figure 2; as can be seen, the relaxation function decays within about 10 seconds from its instantaneous level $E_{0}$ to (almost) its longtime value of $E_{\infty}$. The memory kernel is negative, and increases in value towards zero from an initial level of $-0.375 \mathrm{~s}^{-1}$. Similar to the behavior of $E(t)$, most of the transition in $R(t)$ occurs within the first 10 seconds.

The plate is supported on a Pasternak foundation with $k=0.15 \mathrm{~N} / \mathrm{mm}^{3}$ and $G=4 \cdot 10^{4} \mathrm{~N} / \mathrm{mm}$ ; it is uniformly loaded over a circular area with $a=150 \mathrm{~mm}$ and $q_{0}=0.60 \mathrm{MPa}$ (refer to Equation (8)). Three separate cases are considered hereafter, for which only the stress variation function $f(t)$ is different. Case I deals with a step function variation, Case II considers a two-second box function variation, and Case III deals with a single $\pi$-seconds long half-sine event. The solution for all three cases was based on $N=5$ and $T=10 \mathrm{~s}$, i.e., an analysis interval of $0 \leq t \leq 10$ seconds (see Equation (14)). 

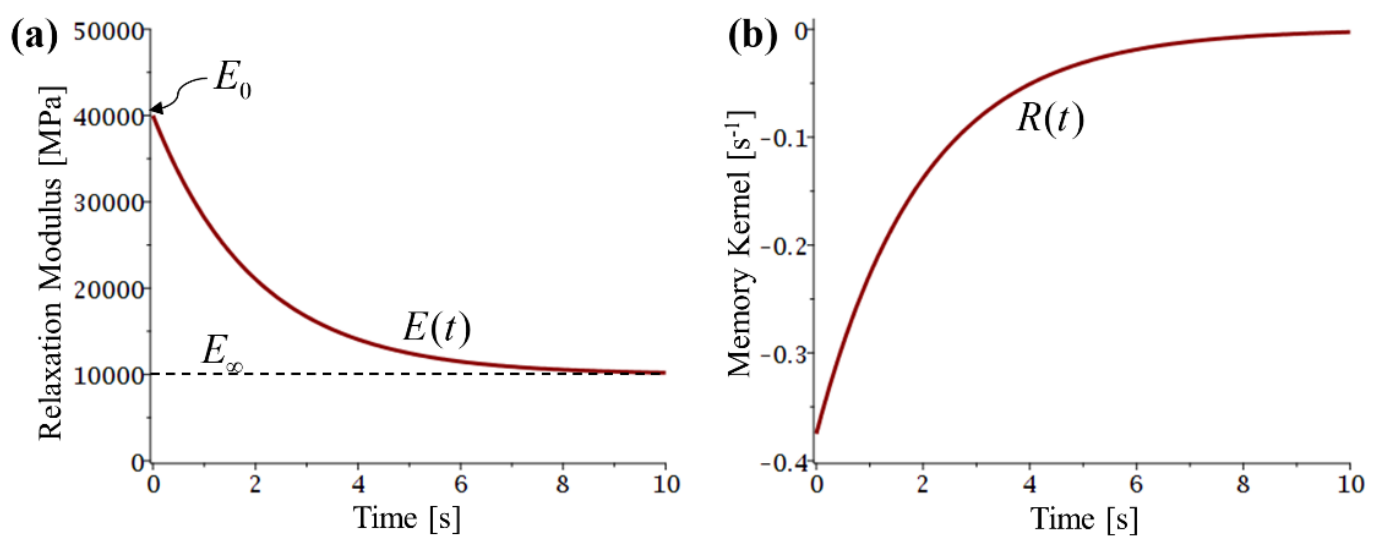

Figure 2. Viscoelastic relaxation function and memory kernel for the illustrative examples.

\subsection{Example cases}

In Case I the stress varies in time according to $f(t)=f_{1}(t)=H(t)$. The iterative functions $f_{2}(t)$ to $f_{6}(t)$ are first constructed based on Equation (9). The outcome is graphically displayed in Figure 3, and the associated mathematical expressions are presented in Appendix B.
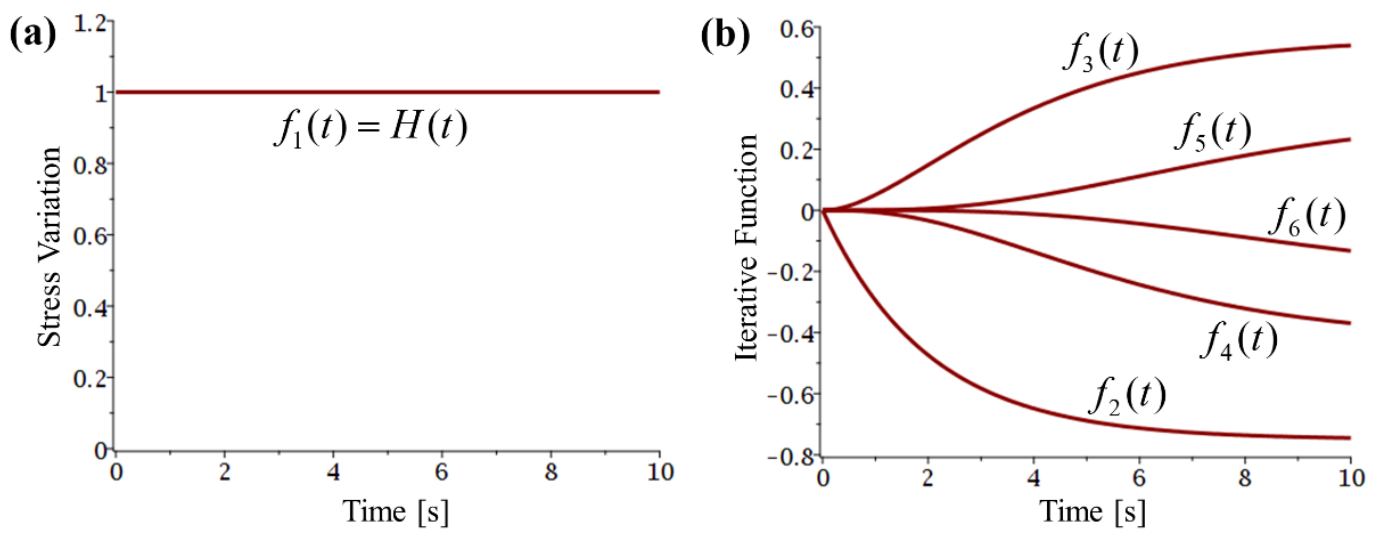

Figure 3. Iterative functions for Case I: (a) stress variation $f(t)=f_{1}(t)$, and (b) iterative functions $f_{2}(t)$ to $f_{6}(t)$.

By means of the least squares formulation in Equations (14) and (15), the function $f_{6}(t)$ was represented as a linear combination of $f_{2}(t)$ to $f_{5}(t)$. Figure 4 displays a magnified view of the function $f_{6}(t)$ as well the error variation function $\operatorname{err}_{5}(t)$ (dashed line). The associated constants $c_{2}$ to $c_{5}$ are also included in the Figure. As can be graphically observed, the error variation is nearly zero across the entire analysis interval indicating a very good representation. 


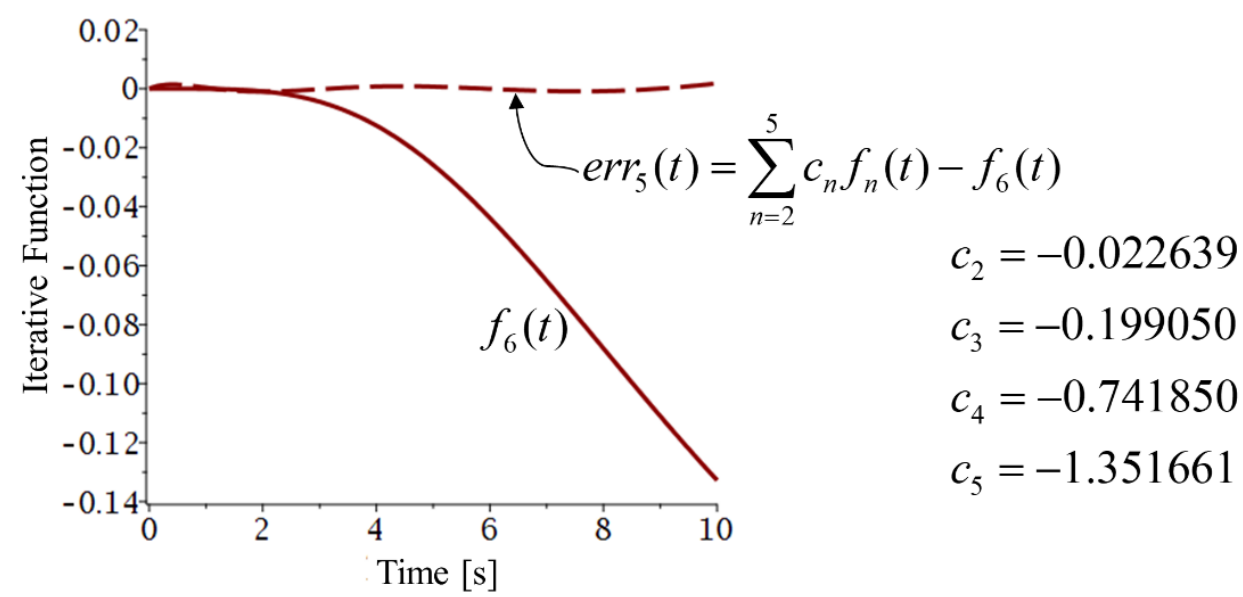

Figure 4. Magnified view of the iterative function $f_{6}(t)$ in Case I (solid line) and the error variation function $\operatorname{err}_{5}(t)$ associated with its representation (dashed line).

With the obtained $c_{n}$ values, the $\mathbf{M}$ matrix was assembled according to Equation (22) and subsequently decomposed according to Equation (25), ultimately allowing Equation (33) to be solved. The related solution parameters are shown in Table 1. Additional computational details are provided in Appendix B.

Table 1. Intermediate solution parameters for Case I example.

\begin{tabular}{|c|c|c|c|c|c|}
\hline$n$ & $l_{n}[\mathrm{~mm}]$ & $b_{n}[-]$ & $\phi_{n}[-]$ & $\alpha_{n}[-]$ & $q_{n}^{*}[\mathrm{MPa}]$ \\
\hline 1 & 656.0 & 0.310 & 0.943 & 0.229 & 49.0 \\
\hline 2 & $612.7+47.9 i$ & $0.349-0.055 i$ & $0.963-0.029 i$ & $0.243-0.019 i$ & $64.8+42.8 i$ \\
\hline 3 & $612.7-47.9 i$ & $0.349+0.055 i$ & $0.963+0.029 i$ & $0.243+0.019 i$ & $64.8-42.8 i$ \\
\hline 4 & $576.9+25.2 i$ & $0.398-0.035 i$ & $0.990-0.019 i$ & $0.259-0.011 i$ & $85.0-105.6 i$ \\
\hline 5 & $576.9-25.2 i$ & $0.398+0.035 i$ & $0.990+0.019 i$ & $0.259+0.011 i$ & $85.0+105.6 i$ \\
\hline
\end{tabular}

Figure 5 graphically presents the viscoelastic solution $w_{a}^{v e}(r, t)$ for Case I. Figure 5a displays the deflection time-history for four different radial offsets, and Figure 5b shows the deflection bowl for $0 \leq r \leq 20 a$ at three different times. It may be observed that shortly after the initial loading is applied the deflections closer to the load center increase with time, while the deflections at larger distances exhibit a small decrease in magnitude.

For Case II, the applied stress was chosen to vary in time according to $f(t)=f_{1}(t)=1-H(t-2)$; as shown in Figure 6a. The corresponding iterative functions $f_{2}(t)$ to $f_{6}(t)$ are graphically displayed in Figure $6 \mathrm{~b}$. The error variation function associated with representing $f_{6}(t)$ 
as a linear combination of $f_{2}(t)$ to $f_{5}(t)$ is displayed in Figure 6c (dashed line). Finally, the viscoelastic solution for the first ten seconds, and at four different radial distances, is shown in Figure 6d. Additional calculation details are provided in Appendix B. Another means of arriving at this solution could have been to utilize linearity, and superpose two Case I results that are delayed in time with opposing sign.
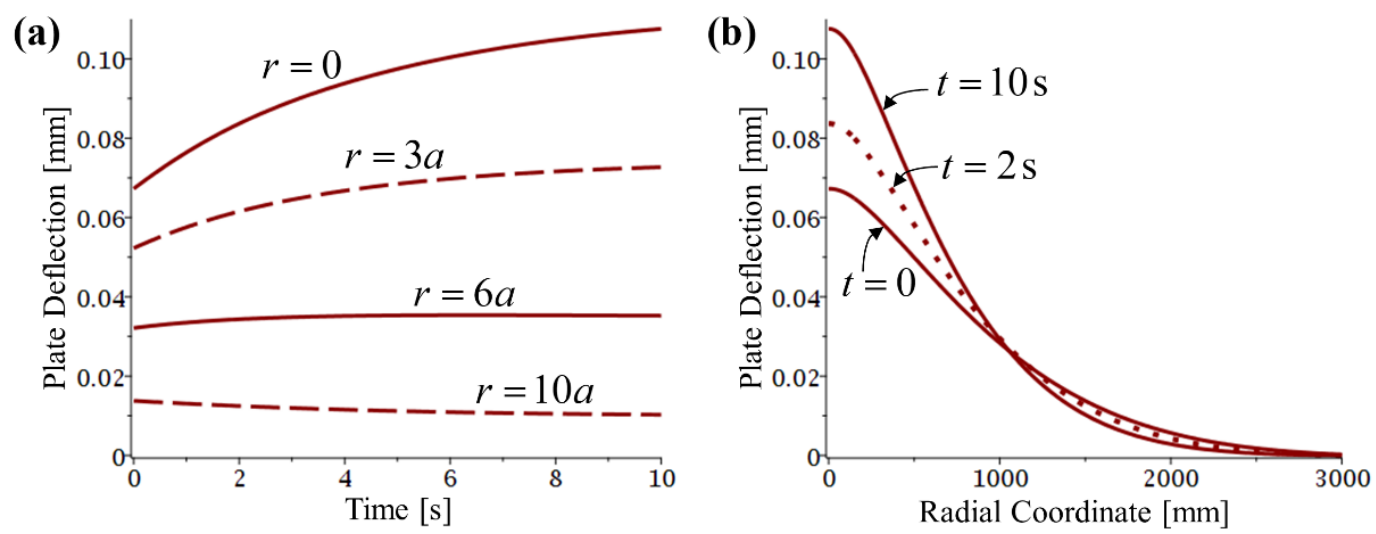

Figure 5. Response of a viscoelastic plate to Case I loading history: (a) deflection time-history at different radial offsets, and (b) deflection bowl at different analysis times.
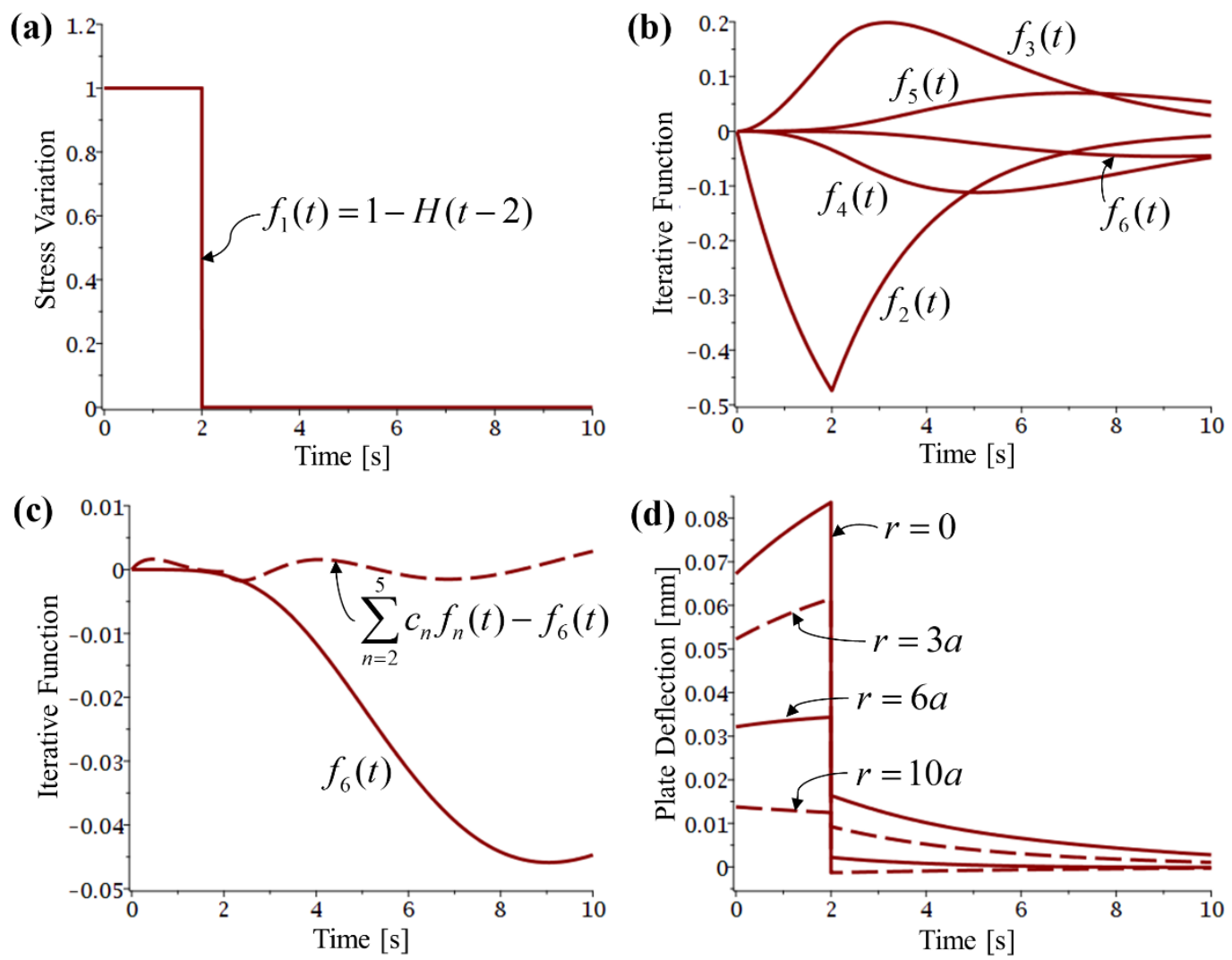

Figure 6. Response of a viscoelastic plate to Case II loading history: (a) applied stress variation, (b) iterative functions $f_{2}(t)$ to $f_{6}(t)$, (c) magnified view of the iterative function $f_{6}(t)$ and error variation 
function $\operatorname{err}_{5}(t)$ associated with its representation (dashed line), and (d) viscoelastic solution for different radial offsets.

For Case III, the applied stress was varied in time according to $f(t)=f_{1}(t)=\sin t-H(t-\pi) \sin t$. This loading is a single half-sine curve as shown in Figure 7a. The related iterative functions $f_{2}(t)$ to $f_{6}(t)$ are graphically displayed in Figure $7 \mathrm{~b}$, the error variation linked to approximating $f_{6}(t)$ is displayed in Figure $7 \mathrm{c}$ (dashed line), and the viscoelastic solution is shown in Figure 7d (refer to Appendix B for more details). An alternative way to arrive at this answer would be to develop a solution for a sine stress variation $f(t)=\sin t$ and another separate solution for a delayed sine load of the form $f(t)=H(t-\pi) \sin (t-\pi)$, and then add the two.
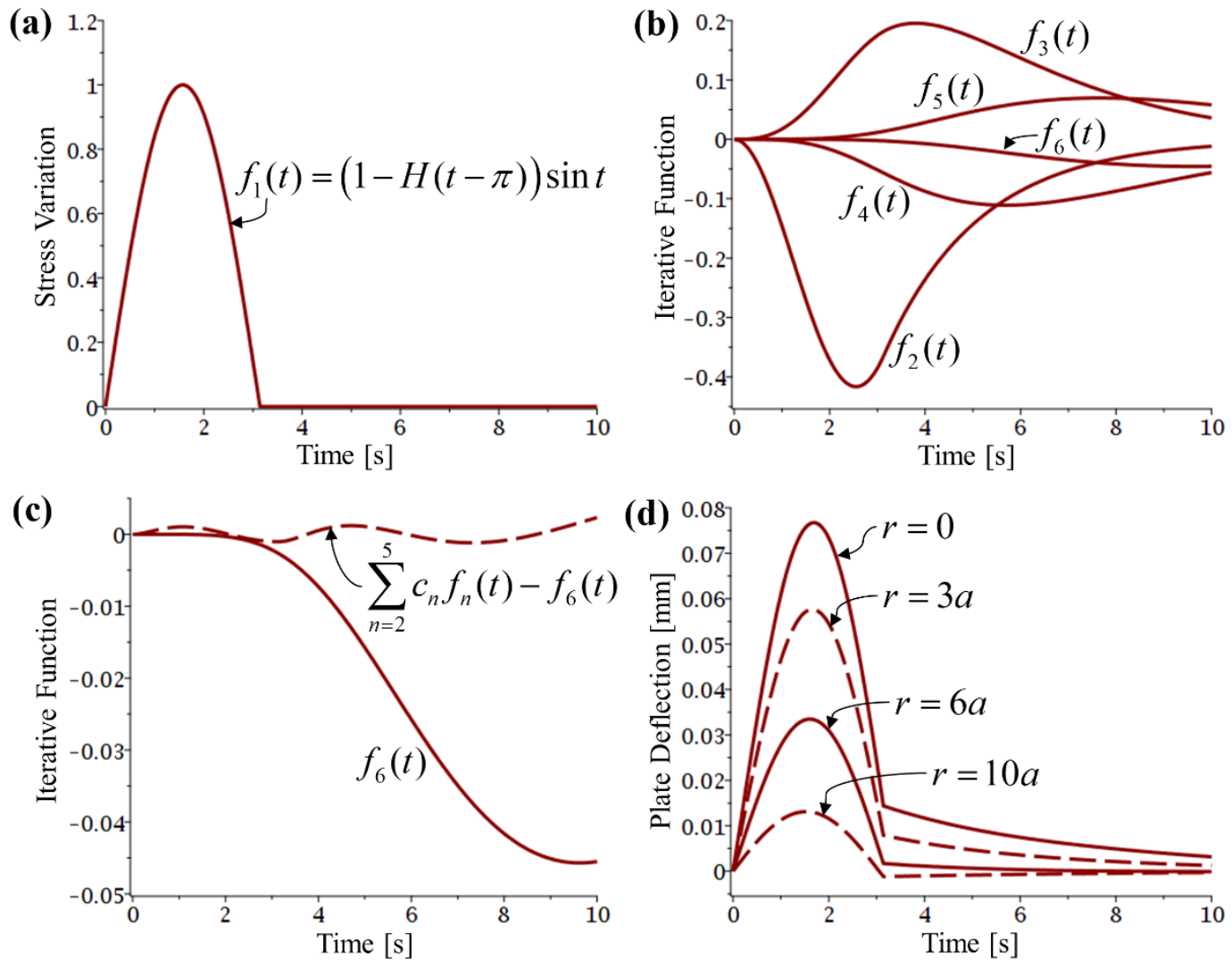

Figure 7. Response of a viscoelastic plate to Case III loading history: (a) applied stress variation, (b) iterative functions $f_{2}(t)$ to $f_{6}(t)$, (c) magnified view of the iterative function $f_{6}(t)$ and error variation function $\operatorname{err}_{5}(t)$ associated with its representation (dashed line), and (d) viscoelastic solution for different radial offsets.

\subsection{Validation}

Validating each of the above-described solutions was performed by substitution into the left-hand-side of Equation (8), and performing analytically all associated differentiation and integration operations. 
If the offered solutions are correct, the outcome in each case should yield the applied stress history for points under the loaded area, and zero for all points outside the loaded area (at all times). Figure 8 displays the outcome from these calculations; Case I is shown in Figure 8a, Case II in Figure 8b, and Case III in Figure 8c. As can be graphically observed, the solutions are correct, given that the applied stress history is essentially reproduced.
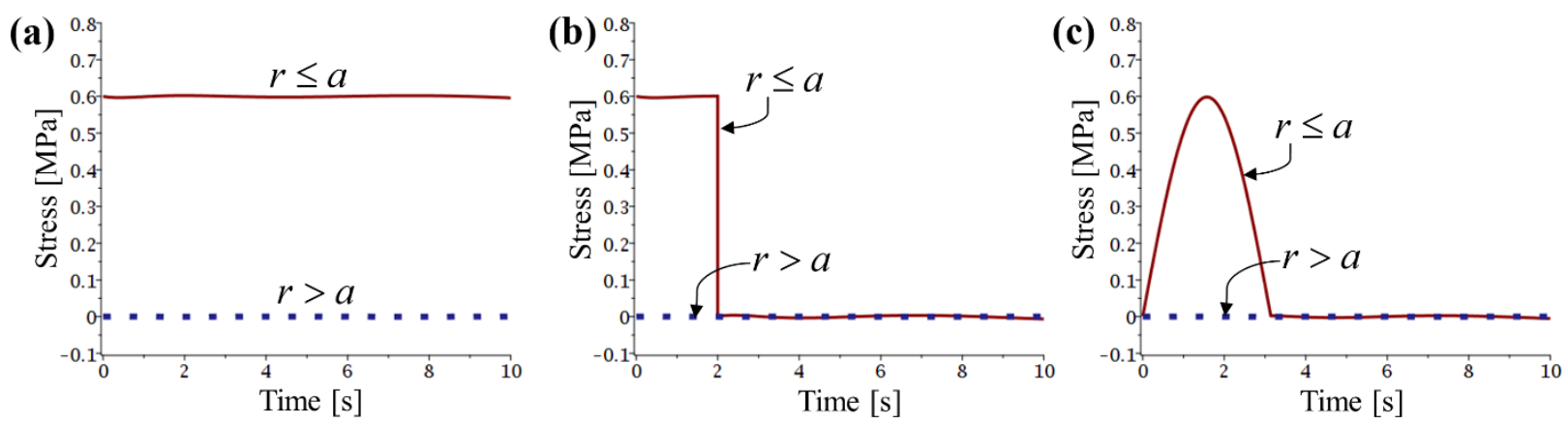

Figure 8. Validation of viscoelastic solutions for $T=10 \mathrm{~s}$ and $N=5$ by means of substitution into the left-hand-side of Equation (8): (a) Case I, (b) Case II, and (c) Case III.

The viscoelastic solution accuracy proposed herein depends on the choice of the total number of iterative functions $N$ in Equation (10). To graphically demonstrate this point, a close view of Case I validation results is offered in Figure 9 for three different $N$ values. Two charts are included for each $N$, depicting calculated stress for $r \leq a$ (solid line) where the target result is $q_{0}=0.6 \mathrm{MPa}$ (indicated by a dashed line), and depicting calculated stress for $r>a$ (dotted line) where the target result is zero (solid line). As expected, increasing $N$ translates into an improved accuracy. What should be noted is the considerable leaps in accuracy upon slight increases in $N$. It may also be observed that even three iterative functions can lead to an acceptable solution. 

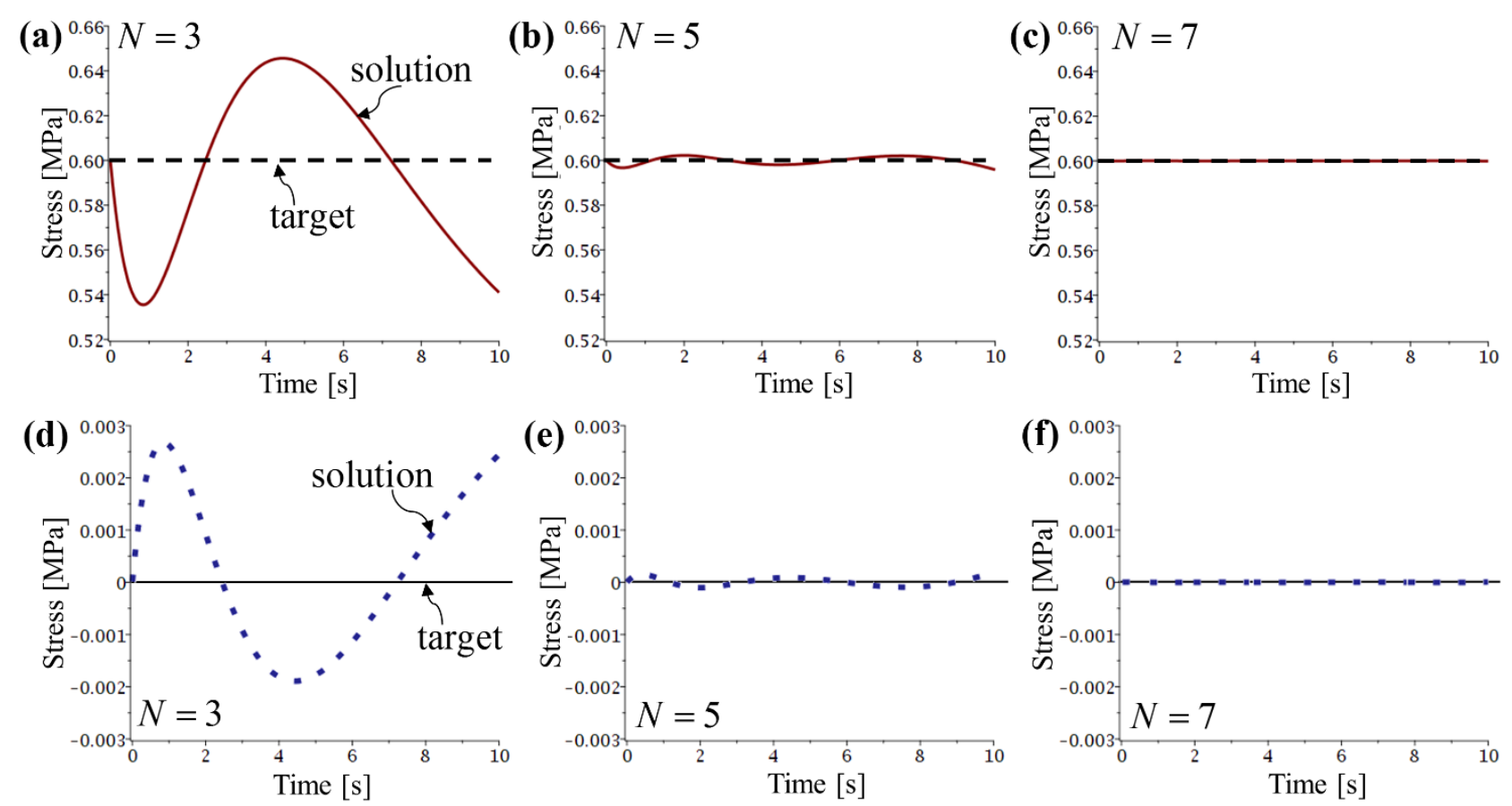

Figure 9. Demonstrating influence of chosen number of iterative functions on Case I solution accuracy; charts (a)-(c) depict validation results under the load, and charts (d)-(f) depict validation results outside the loaded area.

In general terms, the choice of $N$ is case specific, depending on all problem parameters, and particularly on the applied loading variation $f(t)$, the shape of the viscoelastic relaxation function $E(t)$, and the analysis duration for which the solution is sought $T$. For a given set of problem parameters, this choice controls the solution accuracy because it governs the ability of the iterative functions $f_{2}(t) \ldots f_{N}(t)$ to correctly represent $f_{N+1}(t)$ as required by Equation (13). The inaccuracy of this representation is given by Err in Equation (14) after substituting the related $c_{n}$ values; Err should equal zero in the case of perfect representation (i.e., as $N \rightarrow \infty$ ).

The Err values associated with the three example cases are listed in Table 2 for $N$ values in the range of 2 to 7 . It can be clearly seen that as $N$ increases $E r r$ decreases towards zero with an accelerating logarithmic rate. For example, the improvement in Err attained between $N=2$ and $N=3$ is about one order of magnitude, while between $N=6$ and $N=7$ the value of $E r r$ improves by almost two orders of magnitude. This behavior is referred to as super-convergence; it indicates that a very small set of elastic solutions is needed for generating a highly accurate viscoelastic result. In practice, the value of $N$ can be chosen by substituting a solution to the original equation and judging 
the mismatch with the applied stress - as done in Figure 9. Another option is to solve a problem with increasing $N$ values until the changes in the solution becomes meaningless.

Table 2. The inaccuracy of representing $f_{N+1}(t)$ as a linear combination of its predecessors for different $N$ values.

\begin{tabular}{|c|c|c|c|}
\hline & \multicolumn{3}{|c|}{ Value of Err in Equation (14) } \\
\hline$N$ & Case I & Case II & Case III \\
\hline 2 & $6.705 \cdot 10^{-2}$ & $5.279 \cdot 10^{-2}$ & $4.848 \cdot 10^{-2}$ \\
\hline 3 & $3.829 \cdot 10^{-3}$ & $5.793 \cdot 10^{-3}$ & $4.819 \cdot 10^{-3}$ \\
\hline 4 & $1.707 \cdot 10^{-4}$ & $3.879 \cdot 10^{-4}$ & $2.658 \cdot 10^{-4}$ \\
\hline 5 & $5.412 \cdot 10^{-6}$ & $1.415 \cdot 10^{-5}$ & $7.686 \cdot 10^{-6}$ \\
\hline 6 & $1.242 \cdot 10^{-7}$ & $3.148 \cdot 10^{-7}$ & $1.320 \cdot 10^{-7}$ \\
\hline 7 & $2.136 \cdot 10^{-9}$ & $4.777 \cdot 10^{-9}$ & $1.481 \cdot 10^{-9}$ \\
\hline
\end{tabular}

\section{Summary and remarks}

This work addressed the problem of a continuously supported viscoelastic plate of infinite size that is loaded by a distributed stress with time varying intensity. It offered an analytical solution to the governing integro-differential equation, Equation (8). This equation is founded on the assumption of linearity, i.e., that both the foundation response and the plate properties obey the principle of superposition. The derivation was presented in detail, starting from a solution to the elastic problem, and extending to the sought time-dependent case. Associated calculations were demonstrated and validated over three specific cases.

The solution offered herein did not rely on transform techniques but rather on the iterative construction of a set of functions that embody the loading time-variation and the plate's relaxation modulus. A similar approach has been used by Volterra for solving integro-differential equations (Volterra, 1959). However, Volterra's approach required an infinite series of iterative functions, whereas in this study a series of finite length was employed. Following Zevin (1971), the last iterative function was approximated by a linear combination of the preceding iterative functions on a given time interval. Doing so enabled the decomposition of the original viscoelastic problem into a set of independent elastic plate problems, with fictitious elastic properties. The elastic solution set was then carefully assembled to arrive at the sought viscoelastic answer. Ultimately, only a very small number 
of elastic solutions, solved in any order, was needed for generating a highly accurate viscoelastic solution. Thus, the current method is fundamentally different from the incremental time-stepping approach traditionally utilized by numerical solvers when handling linear viscoelasticity (e.g., Taylor, Pister, \& Goudreau, 1970; Zocher, Groves, \& Allen, 1997).

The proposed method is deemed well suited for engineering calculations given that it is naturally equipped to receive as input realistic viscoelastic material properties expressed as a multiterm Prony series (Williams, 1964)

$$
E(t)=E_{\infty}+\sum_{j} E_{j} e^{-t / \rho_{j}}
$$

wherein the $E_{j}$ 's are relaxation strengths and the $\rho_{j}$ 's are relaxation times. Also, due to its closedform nature, the solution is deemed well suited for inverse analysis schemes wherein model responses are evaluated many times over (with different parameters) in search for convergence. Such computational efficiency is especially relevant for processing the huge amounts of deflection data collected by modern moving measurement platforms (Andersen, Levenberg, \& Andersen 2018). In this connection, backcalculation of deflection measurements offers a practical method for quantifying the governing Pasternak foundation parameters $k$ and $G$ as well as the governing relaxation function $E(t)$. Finally, due to the high accuracy of the new solution it can serve as a reference for benchmarking numerical solvers.

\section{Acknowledgements}

This work was supported in part by the 2015 J.S. Braun/Braun Intertec professorship grant (College of Science and Engineering, University of Minnesota).

\section{References}

Altenbach, H., \& Eremeyev, V. A. (2009). On the bending of viscoelastic plates made of polymer foams. Acta Mechanica, 204, 137-154.

Andersen, S., Levenberg, E., \& Andersen, M. B. (2018). Efficient reevaluation of surface displacements in a layered elastic half-space. International Journal of Pavement Engineering, DOI: $10.1080 / 10298436.2018 .1483502$.

Anderton, G. L. (2000). Engineering properties of resin modified pavement (RMP) for mechanistic design. Report ERDC/GLTR-00-2, US Army Corps of Engineers, Engineer Research and Development Center. 
Brotchie, J. F., \& Silvester, R. (1969). On crustal flexure. Journal of Geophysical Research, 74(22), 5240-5252.

Elattary, M.A., Cozzarelli, F. A., \& Shaw, R. P. (1988). Stress intensification in a viscoelastic plate on a Winkler foundation and under a large thermal gradient. Acta Mechanica, 72, 189-203.

Filonenko-Borodich, M. M. (1940). Some approximate theories of elastic foundation. Scientific Notes of the Moscow State University: Mechanics, 46, 3-18 (in Russian).

Flügge, W. (1975). Viscoelasticity, 2nd Edition. Springer-Verlag Berlin Heidelberg GmbH.

Fwa, T. F., Shi, X. P., \& Tan, S. A. (1996). Use of Pasternak foundation model in concrete pavement analysis. Journal of Transportation Engineering, 122(4), 323-328.

Hemsley, J. A. (Ed.). (2000). Design applications of raft foundations. Thomas Telford.

Hetenyi, M. (1946). Beams on elastic foundation: theory with applications in the fields of civil and mechanical engineering. University of Michigan Press, Ann Arbor, Michigan.

Hewitt, J. S., \& Mazumdar, J. (1974). Vibration of viscoelastic plates under transverse load by the method of constant deflection contours. Journal of Sound and Vibration, 33(3), 319-333.

Hosking, R. J., Sneyd, A. D., \& Waugh, D. W. (1988). Viscoelastic response of a floating ice plate to a steadily moving load. Journal of Fluid Mechanics, 196, 409-430.

Hui, D. (1986). Viscoelastic response of floating ice plates under distributed or concentrated loads. Journal of Strain Analysis, 21(3), 135-143.

Khazanovich, L. \& Ioannides, A. M. (1993). Finite element analysis of slabs-on-grade using higher order subgrade soil models. Proceedings of the Airfield Specialty Conference, Airport Pavement Innovations: Theory to Practice, Vicksburg, MS, pp. 16-30.

Kerr, A. D., \& El-Sibaie, M. A. (1989). Green's functions for continuously supported plates. Journal of Applied Mathematics and Physics (ZAMP), 40, 15-38.

Kerr, A. D. (1964). Elastic and viscoelastic foundation models. Journal of Applied Mechanics, 31(3), 491-498

Kerr, A. D. (1965). Continuity in foundation models and related problems. Research Report 109, DA Task IV025001A13001, Cold Regions Research and Engineering Laboratory (CRREL), Hanover, New Hampshire, USA.

Kerr, A. D., \& Palmer, W. T. (1972). The deformations and stresses in floating ice plates. Acta Mechanica, 15, 57-72.

Korenev, B. G. (1954). Problems of analysis of beams and plates on elastic foundation. Gosudarstvennoe Izdatel' stvo Literatury po Stroitel' stvu i Arkhitekture, Moscow (in Russian).

Louhghalam, A., Akbarian, M., \& Ulm, F. J. (2013). Flügge's conjecture: Dissipation- versus deflection-induced pavement-vehicle interactions. Journal of Engineering Mechanics, 140(8), 04014053(1-10).

Marvin, E. L. (1972). Viscoelastic plate on poroelastic foundation. Journal of the Engineering Mechanics Division, 98(4), 911-928.

Mase, G. E. (1961). Behavior of viscoelastic plates in bending. Transactions of the American Society of Civil Engineers, 126(1), 1569-1582.

Misra, J. C., \& Samanta, S. (1980). Bending of a heated viscoelastic plate on an elastic foundation. Journal of Non-Equilibrium Thermodynamics, 5(3), 137-142.

Pister, K. S. (1961). Viscoelastic plate on a viscoelastic foundation. Journal of the Engineering Mechanics Division, 87(1), 43-54.

Pister, K. S., \& Monismith, C. L. (1960). Analysis of visco-elastic flexible pavements. Highway Research Board Bulletin, 269, 1-15. 
Pister, K. S., \& Westmann, R. A., (1962). Analysis of viscoelastic pavements subjected to moving loads. Proceedings of the First International Conference on the Structural Design of Asphalt Pavements, Ann Arbor, Michigan, pp. 522-529;

Pożarycki, A., Fengier, J., Górnaś, P., \& Wanatowski, D. (2018). Backcalculation of pavements incorporating Grouted Macadam technology. Road Materials and Pavement Design, 19(6), $1372-1388$.

Pronk, A. C. (1993). The Pasternak foundation: an attractive alternative for the Winkler foundation. Proceedings of the Fifth International Conference on Concrete Pavement Design and Rehabilitation Volume 1, pp. 349-357, The international Society for Concrete Pavement (ISCP).

Radovskii, B. S. (1979). Application of the calculation scheme for a layered viscoelastic medium to the estimation of the stressed state of highways and airport pavements with moving loads. Soviet Applied Mechanics, 15(10), 940-946.

Reissner, E. (1958). A note on deflection of plates on viscoelastic foundation. Journal of Applied Mechanics, 25, 144-145.

Robertson, S. R. (1971). Solving the problem of forced motion of viscoelastic plates by Valanis' method with an application to a circular plate. Journal of Sound and Vibration, 14(3), 263-278.

Selvadurai, A. P. S. (1979). Elastic analysis of soil-foundation interaction. Developments in Geotechnical Engineering, 17, Elsevier/North-Holland Inc., New York, NY.

Selvadurai, A. P. S. (2007). The analytical method in geomechanics. Applied Mechanics Reviews, 60, 87-106.

Shu, X., \& Huang, B. (2014). Recycling of waste tire rubber in asphalt and Portland cement concrete: an overview. Construction and Building Materials, 67, 217-224.

Smith, T. L. (1971). Empirical equations for representing viscoelastic functions and for deriving spectra. Journal of Polymer Science Part C, 35, 39-50.

Takagi, S. (1978). The viscoelastic deflection of an infinite floating ice plate subjected to a circular load. CRREL Report 78-5, U.S. Army Cold Regions Research and Engineering Laboratory, Hanover, New Hampshire.

Taylor, R. L., Pister, K. S., \& Goudreau, G. L. (1970). Thermomechanical analysis of viscoelastic solids. International Journal for Numerical Methods In Engineering, 2, 45-59.

Timoshenko, S.P. \& Woinowsky-Krieger, S. (1959). Theory of plates and shells, 2nd Ed. McGrawHill, New York, NY.

Van Cauwelaert, F., Stet, M., \& Jasienski, A. (2002). The general solution for a slab subjected to centre and edge loads and resting on a Kerr foundation. International Journal of Pavement Engineering, 3(1), 1-18.

Vlasov, V. Z., \& Leont'ev, N. N. (1966). Beams, plates and shells on elastic foundations," (translated from Russian), Israel Program for Scientific Translations Ltd, Catalogue, 1453.

Volterra, V. (1959). Theory of functionals and of integral and integro-differential equations. New York, NY: Dover, p. 226.

Von Kármán, T., \& Biot, M. A. (1940). Mathematical methods in engineering: an introduction to the mathematical treatment of engineering problems. McGraw-Hill book company Inc., p. 505.

Watson, G. N. (1944). A treatise on the theory of Bessel functions, 2nd Edition. Cambridge University Press, Cambridge, United Kingdom.

Watts, A. B. (2001). Isostasy and flexure of the lithosphere. Cambridge University Press, United Kingdom, Cambridge, p. 458. 
Westergaard, H. M. (1939). Stresses in concrete runways of airports. Proceedings of the Highway Research Board, 19, pp. 197-205.

Westergaard, H. M. (1948). New formulas for stresses in concrete pavements of airfields. Transactions of the American Society of Civil Engineers, 113, 425-444.

Williams, M. L. (1964). Structural analysis of viscoelastic materials. AIAA Journal, 2(5), 785-808.

Wyman, M. (1950). Deflections of an infinite plate. Canadian Journal of Research, 28, 293-302.

Zevin, A. A. (1971). Approximation of functions of integral operators in hereditary theories of elasticity and aging (translated to English by the Central Scientific-Research Institute of Steel Structural Planning, Leningrad). Prikladnaya Mekhanika, 7(11), 90-95.

Zocher, M. A., Groves, S. E., \& Allen, D. H. (1997). A three-dimensional finite element formulation for thermoviscoelastic orthotropic media. International Journal of Numerical Methods in Engineering, 40, 2267-2288. 


\section{Appendix A: The elastic problem}

This Appendix is dedicated to the rederivation of Equation (2) following Korenev (1954). The starting point is the governing differential equation for an infinite Germain-Kirchhoff plate (Timoshenko \& Woinowsky-Krieger, 1959) supported on a Pasternak foundation under axisymmetric loading conditions

$$
D \nabla^{4} w_{z}(r)-G \nabla^{2} w_{z}(r)+k w_{z}(r)=q_{z}(r)
$$

where $w_{z}(r)$ denotes the deflection at a radial coordinate $r$, and $q_{z}(r)$ denotes the intensity of the applied vertical stress. To facilitate the derivation, this Equation is written in an equivalent form

$$
\nabla_{s}^{4} w(s)-2 b \nabla_{s}^{2} w(s)+w(s)=\frac{q(s)}{k}
$$

where the sought after plate deflection is $w(s)=w_{z}(s l)$, the applied loading is $q(s)=q_{z}(s l)$, and the subscript $s$ in $\nabla_{s}^{4}$ and $\nabla_{s}^{2}$ indicates that the Laplacians are applied with respect to $s$ instead of $r$.

A general homogenous solution to Equation (A.2) is $w_{0}=w_{0}(s)$, given by the following expression (Vlasov \& Leont'ev, 1966; Watson, 1944)

$$
w_{0}=a_{1} J_{0}\left(s \sqrt{\xi_{1}}\right)+a_{2} Y_{0}\left(s \sqrt{\xi_{1}}\right)+a_{3} J_{0}\left(s \sqrt{\xi_{2}}\right)+a_{4} Y_{0}\left(s \sqrt{\xi_{2}}\right)
$$

where $Y_{0}$ is the Bessel function of the second kind of order zero (Von Kármán \& Biot, 1940), $\xi_{1,2}=-b \pm \sqrt{b^{2}-1}$, and $a_{1}, \ldots, a_{4}$ are constants to be determined from enforcing boundary conditions. Without loss of generality, the case of $b \leq 1$ is considered hereafter from which $\xi_{1,2}=-b \pm i \sqrt{1-b^{2}}$. Transferring Equation (A.3) into a polar representation for $\xi_{1,2}$ gives

$$
w_{0}=a_{1} J_{0}\left(s e^{i \phi}\right)+a_{2} Y_{0}\left(s e^{i \phi}\right)+a_{3} J_{0}\left(s e^{-i \phi}\right)+a_{4} Y_{0}\left(s e^{-i \phi}\right)
$$

where $\phi$ is defined in Equation (3).

Now, using the definition $H_{0}^{(1)}(\cdot)=J_{0}(\cdot)+i Y_{0}(\cdot)$ and the definition $H_{0}^{(2)}(\cdot)=J_{0}(\cdot)-i Y_{0}(\cdot)$, Equation (A.4) becomes

$$
w_{0}=A_{1} J_{0}\left(s e^{i \phi}\right)+A_{2} H_{0}^{(1)}\left(s e^{i \phi}\right)+A_{3} J_{0}\left(s e^{-i \phi}\right)+A_{4} H_{0}^{(2)}\left(s e^{-i \phi}\right)
$$

with $A_{1}=a_{1}+i a_{2}, A_{2}=-i a_{2}, A_{3}=a_{3}-i a_{4}$, and $A_{4}=i a_{4}$. 
Equation (A.5) may serve as basis for expressing the solution of a plate loaded by a concentrated force with intensity $P$ acting at $s=0$. To achieve this the homogenous solution must be adjusted to yield a deflection that is real, that vanishes as $s \rightarrow \infty$, and that is finite with zero slope at $s=0$. Meeting all these conditions is assured when $A_{1}=A_{3}=0$ and $A_{2}=A_{4}$, reducing Equation (A.5) to

$$
w_{0}=A H_{0}^{(1)}\left(s e^{i \phi}\right)+A H_{0}^{(2)}\left(s e^{-i \phi}\right)
$$

with $A$ being a real coefficient determined from boundary conditions.

As means of finding $A$, vertical shear stresses are introduced inside the plate with an overall intensity equivalent to $P$, as follows

$$
P=\lim _{s \rightarrow 0} \int_{\theta=0}^{2 \pi} \frac{D}{l^{3}} \frac{d\left(\nabla_{s}^{2} w_{0}\right)}{d s} s l d \theta=\lim _{s \rightarrow 0} \frac{2 \pi s D}{l^{2}} \frac{d\left(\nabla_{s}^{2} w_{0}\right)}{d s}=\frac{2 \pi D}{l^{2}} \lim _{s \rightarrow 0} \frac{s d\left(\nabla_{s}^{2} w_{0}\right)}{d s}
$$

where it can be seen that the shear stresses are integrated circumferentially around, and in close proximity to, the origin of the radial coordinate. Applying a Taylor series expansion about $s=0$ to Equation (A.6) gives

$$
\lim _{s \rightarrow 0}\left(A H_{0}^{(1)}\left(s e^{i \phi}\right)+A H_{0}^{(2)}\left(s e^{-i \phi}\right)\right) \approx A\left(\frac{s^{2} \ln s}{\pi} \sin 2 \phi+2-\frac{4 \phi}{\pi}\right)
$$

from which it is straightforward to derive

$$
\lim _{s \rightarrow 0} \frac{s d\left(\nabla_{s}^{2} w_{0}\right)}{d s}=\frac{4 A}{\pi} \sin 2 \phi
$$

and by virtue of Equation (A.7)

$$
A=\frac{P l^{2}}{8 D \sin 2 \phi}=\frac{P}{8 k l^{2} \sin 2 \phi}
$$

Hence, the plate deflection for a concentrated force $w_{P}=w_{P}(s)$ is

$$
w_{P}=\frac{P}{8 k l^{2} \sin 2 \phi}\left(H_{0}^{(1)}\left(s e^{i \phi}\right)+H_{0}^{(2)}\left(s e^{-i \phi}\right)\right)
$$

Figure A1 graphically presents the outcome of this solution in a non-dimensional (normalized) form, with $w_{P} k l^{2} / P$ as the ordinate and $s$ as the abscissa. Several curves are plotted, each associated 
with a different value of $b$ in the range of 0 to 1 . The case of $b=0$ represents the solution for an elastic plate supported by a Winkler foundation, for which Equation (A.11) reduces to $w_{P}=-P\left(2 \pi k l^{2}\right)^{-1} \operatorname{kei}_{0}(s)$ with $\operatorname{kei}_{0}(\cdot)$ as the Kelvin kei function of order zero (Wyman, 1950; Westergaard, 1939).

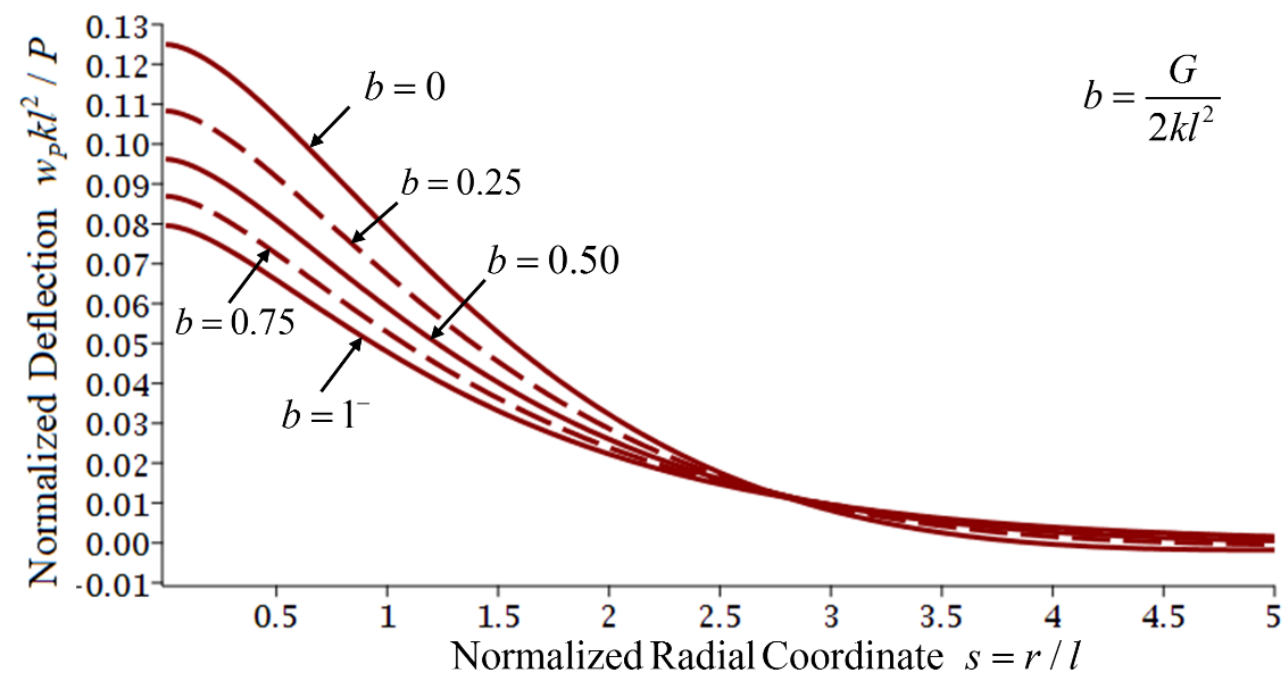

Figure A1. Deflection of an elastic plate on a Pasternak foundation loaded by a point force, Equation (A.11).

The point force solution $w_{P}$ in Equation (A.11) is now extended to the case of a ring load $w_{\beta}=w_{\beta}(s)$, i.e., the deflection due to a vertical line stress acting over a circle. Figure A2 graphically displays the associated superposition wherein the intensity of the ring loading is $q_{\beta}$ (units of force/length), the ring radius is $\beta l$, and a central angle $\theta$ is introduced to denote the position of an infinitesimal ring segment with length $\beta l d \theta$ exerting a point force of intensity $q_{\beta} \beta l d \theta$. As indicated in this Figure, the normalized distance between the point of calculation and any ring segment is $\sqrt{s^{2}+\beta^{2}-2 s \beta \cos \theta}$. Accordingly, and based on Equation (A.11), the sought deflection expression is obtained by integrating over the central angle of the ring, collecting the contribution from all infinitesimally loaded ring segments

$$
w_{\beta}=\frac{\beta q_{\beta}}{8 k l \sin 2 \phi} \int_{0}^{2 \pi}\left(\begin{array}{l}
H_{0}^{(1)}\left(e^{i \phi} \sqrt{s^{2}+\beta^{2}-2 s \beta \cos \theta}\right) \\
+H_{0}^{(2)}\left(e^{-i \phi} \sqrt{s^{2}+\beta^{2}-2 s \beta \cos \theta}\right)
\end{array}\right) d \theta
$$

The integration in Equation (A.12) can be carried out analytically, giving the following solution 


$$
w_{\beta}=\left\{\begin{array}{l}
s \leq \beta, \frac{\pi \beta q_{\beta}}{4 k l \sin 2 \phi}\left(H_{0}^{(1)}\left(\beta e^{i \phi}\right) J_{0}\left(s e^{i \phi}\right)+H_{0}^{(2)}\left(\beta e^{-i \phi}\right) J_{0}\left(s e^{-i \phi}\right)\right) \\
s>\beta, \frac{\pi \beta q_{\beta}}{4 k l \sin 2 \phi}\left(H_{0}^{(1)}\left(s e^{i \phi}\right) J_{0}\left(\beta e^{i \phi}\right)+H_{0}^{(2)}\left(s e^{-i \phi}\right) J_{0}\left(\beta e^{-i \phi}\right)\right)
\end{array}\right.
$$

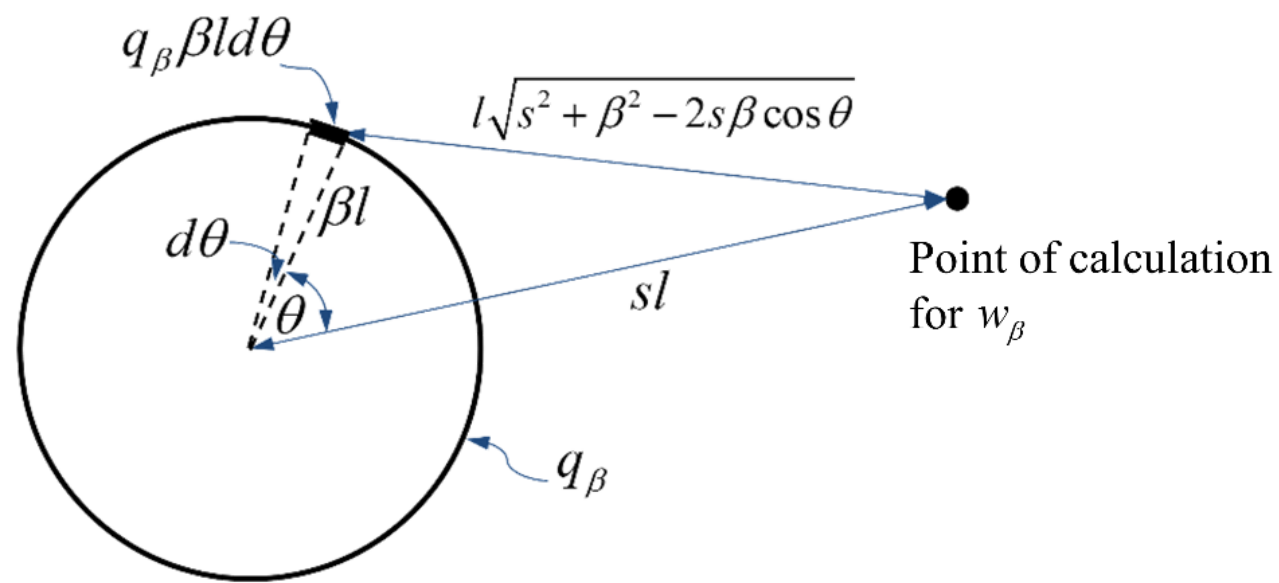

Figure A2. Superposition for extending a point force solution to a ring load case.

The ring load solution $w_{\beta}$ is further extended for calculating the plate deflection $w_{\alpha}=w_{\alpha}(s)$ due to a uniform vertical stress acting over a circular area. The stress intensity is $q_{\alpha}$ (units of force/length ${ }^{2}$ ) and the circle radius is $\alpha l$. For this purpose $q_{\beta}$ in Equation (A.13) is replaced with $q_{\alpha} l d \beta$, and an integration is carried out with respect to $\beta$ from zero to $\alpha$; the outcome is

$$
w_{\alpha}=\left\{\begin{array}{l}
s \leq \alpha, \frac{\pi \alpha q_{\alpha}}{4 k \sin 2 \phi}\left(\begin{array}{l}
\frac{2}{\pi \alpha}\left(i e^{-2 i \phi}-i e^{2 i \phi}\right)+e^{-i \phi} H_{1}^{(1)}\left(\alpha e^{i \phi}\right) J_{0}\left(s e^{i \phi}\right) \\
+e^{i \phi} H_{1}^{(2)}\left(\alpha e^{-i \phi}\right) J_{0}\left(s e^{-i \phi}\right)
\end{array}\right) \\
s>\alpha, \frac{\pi \alpha q_{\alpha}}{4 k \sin 2 \phi}\left(e^{-i \phi} H_{0}^{(1)}\left(s e^{i \phi}\right) J_{1}\left(\alpha e^{i \phi}\right)+e^{i \phi} H_{0}^{(2)}\left(s e^{-i \phi}\right) J_{1}\left(\alpha e^{-i \phi}\right)\right)
\end{array}\right.
$$

This expression is identical to Equation (2) considering that $w_{a}(r)=w_{\alpha}(r / l)$. It is noted that there exists a different expression for $w_{\alpha}$ which is semi-analytical (Van Cauwelaert, Stet, \& Jasienski, 2002)

$$
w_{\alpha}=\frac{\alpha q_{\alpha}}{k} \int_{0}^{\infty} \frac{J_{0}(m s) J_{1}(m \alpha)}{m^{4}+2 b m^{2}+1} d m
$$


where $b$ is not limited to reside between zero and unity, and there is no need to distinguish between $s \leq \alpha$ or $s>\alpha$. However, this expression must be evaluated numerically and its form does not lend itself to be further extended to the case of a viscoelastic plate. 


\section{Appendix B: Calculation details for the example cases}

\section{$\underline{\text { Case I calculation details }}$}

Analytic expressions for the iterative functions depicted in Figure 3 are

$$
\begin{aligned}
& f_{1}(t)=H(t) \\
& f_{2}(t)=\frac{3}{4}\left(e^{-t / 2}-1\right) \\
& f_{3}(t)=\frac{9 e^{-t / 2}}{32}\left(2 e^{t / 2}-t-2\right) \\
& f_{4}(t)=\frac{27 e^{-t / 2}}{512}\left(t^{2}+4 t+8-8 e^{t / 2}\right) \\
& f_{5}(t)=\frac{27 e^{-t / 2}}{4096}\left(48 e^{t / 2}-t^{3}-6 t^{2}-24 t-48\right) \\
& f_{6}(t)=\frac{81 e^{-t / 2}}{131072}\left(t^{4}+8 t^{3}+48 t^{2}+192 t+384-384 e^{t / 2}\right)
\end{aligned}
$$

The M Matrix, Equation (22), and its decomposition, Equation (25), are

$$
\begin{aligned}
\mathbf{M} & =\left[\begin{array}{ccccc}
1.000 & 0 & 0 & 0 & 0 \\
1.000 & 1.000 & 0 & 0 & -0.022639 \\
0 & 1.000 & 1.000 & 0 & -0.199050 \\
0 & 0 & 1.000 & 1.000 & -0.741850 \\
0 & 0 & 0 & 1.000 & -0.351661
\end{array}\right] \\
\mathbf{A} & =\left[\begin{array}{ccccc}
1.000 & 0 & 0 & 0 & 0 \\
0 & 0.733+0.236 i & 0 & 0 & 0 \\
0 & 0 & 0.733-0.236 i & 0 & 0 \\
0 & 0 & 0 & 0.591+0.104 i & 0 \\
0 & 0 & 0 & 0 & 0.591-0.104 i
\end{array}\right] \\
\mathbf{V} & =\left[\begin{array}{ccccc}
0.122 & 0 & 0 & 0 & 0 \\
0.108 & 0.036+0.020 i & 0.036-0.020 i & -0.037-0.001 i & -0.037+0.001 i \\
0.401 & 0.275+0.067 i & 0.275-0.067 i & -0.243-0.039 i & -0.243+0.039 i \\
0.731 & 0.712 & 0.712 & -0.663-0.073 i & -0.663+0.073 i \\
0.541 & 0.627-0.137 i & 0.627+0.137 i & -0.703 & -0.703
\end{array}\right]
\end{aligned}
$$




$$
\mathbf{V}^{-1}=\left[\begin{array}{ccccc}
81.7 & 0 & 0 & 0 & 0 \\
108+71.3 i & -45.7+6.48 i & 10.7-12.5 i & 0.11+5.87 i & -1.42-1.54 i \\
108-71.3 i & -45.7-6.48 i & 10.7+12.5 i & 0.11-5.87 i & -1.42+1.54 i \\
142-176 i & -39.5+86.8 i & 7.09-39.6 i & 1.24+16.9 i & -2.28-6.79 i \\
142+176 i & -39.5-86.8 i & 7.09+39.6 i & 1.24-16.9 i & -2.28+6.79 i
\end{array}\right]
$$

\section{Case II calculation details}

Analytic expressions for the iterative functions depicted in Figure 6 are

$$
\begin{aligned}
& f_{1}(t)=1-H(t-2) \\
& f_{2}(t)=\frac{3}{4}\left(H(t-2)-H(t-2) \mathrm{e}^{-t / 2+1}+e^{-t / 2}-1\right) \\
& f_{3}(t)=\frac{9}{32}\left(H(t-2) t e^{-t / 2+1}-(t+2) e^{-t / 2}-2 H(t-2)+2\right) \\
& f_{4}(t)=\frac{27}{512}\left(\left(t^{2}+4 t+8\right) e^{-t / 2}-\left(t^{2}+4\right) e^{1-t / 2} H(t-2)+8 H(t-2)-8\right) \\
& f_{5}(t)=\frac{27}{4096}\left(\begin{array}{l}
\left(t^{3}+12 t+16\right) e^{1-t / 2} H(t-2)-\left(t^{3}+6 t^{2}+24 t+48\right) e^{-t / 2} \\
-48 H(t-2)+48
\end{array}\right) \\
& f_{6}(t)=\frac{81}{131072}\left(\begin{array}{l}
\left(t^{4}+8 t^{3}+48 t^{2}+192 t+384\right) e^{-t / 2} \\
-\left(t^{4}+24 t^{2}+64 t+144\right) e^{1-t / 2} H(t-2) \\
+384 H(t-2)-384
\end{array}\right)
\end{aligned}
$$

The M Matrix, Equation (22), and its decomposition, Equation (25), are

$$
\mathbf{M}=\left[\begin{array}{ccccc}
1.000 & 0 & 0 & 0 & 0 \\
1.000 & 1.000 & 0 & 0 & -0.023492 \\
0 & 1.000 & 1.000 & 0 & -0.193315 \\
0 & 0 & 1.000 & 1.000 & -0.720506 \\
0 & 0 & 0 & 1.000 & -0.330980
\end{array}\right]
$$




$$
\begin{aligned}
\mathbf{A} & =\left[\begin{array}{ccccc}
1.000 & 0 & 0 & 0 & 0 \\
0 & 0.774+0.244 i & 0 & 0 & 0 \\
0 & 0 & 0.774-0.244 i & 0 & 0 \\
0 & 0 & 0 & 0.561+0.139 i & 0 \\
0 & 0 & 0 & 0 & 0.561-0.139 i
\end{array}\right] \\
\mathbf{V} & =\left[\begin{array}{ccccc}
-0.013 & 0 & 0 & 0 & 0 \\
-0.106 & 0.037+0.025 i & 0.037-0.025 i & 0.035+0.011 i & 0.035-0.011 i \\
-0.395 & 0.283+0.076 i & 0.283-0.076 i & 0.224+0.045 i & 0.224-0.045 i \\
-0.730 & 0.716 & 0.716 & 0.644+0.100 i & 0.644-0.100 i \\
-0.548 & 0.618-0.136 i & 0.618+0.136 i & 0.722 & 0.722
\end{array}\right]
\end{aligned}
$$

$$
\mathbf{V}^{-1}=\left[\begin{array}{ccccc}
-77.7 & 0 & 0 & 0 & 0 \\
43.5+83.0 i & -30.1-8.18 i & 8.81-5.49 i & -0.66+3.39 i & -0.68-0.93 i \\
43.5-83.0 i & -30.1+8.18 i & 8.81+5.49 i & -0.66-3.39 i & -0.68+0.93 i \\
-82.4+63.9 i & 27.3-39.5 i & -6.50+21.1 i & -0.08-10.2 i & 1.45+4.46 i \\
-83.4-63.9 i & 27.3+39.5 i & -6.50-21.1 i & -0.08+10.2 i & 1.45-4.46 i
\end{array}\right]
$$

\section{Case III calculation details}

Analytic expressions for the iterative functions depicted in Figure 7 are

$$
\begin{aligned}
& f_{1}(t)=(1-H(t-\pi)) \sin t \\
& f_{2}(t)=\frac{3}{20}\left(\begin{array}{l}
(\sin t-2 \cos t) H(t-\pi)-2 e^{\pi / 2-t / 2} H(t-\pi)+2 \cos t \\
-\sin t-2 e^{-t / 2}
\end{array}\right) \\
& f_{3}(t)=\frac{9}{400}\left(\begin{array}{l}
(5 t+4-5 \pi) e^{\pi / 2-t / 2} H(t-\pi)+(4 \cos t+3 \sin t) H(t-\pi) \\
+(5 t+4) e^{-t / 2}-4 \cos t-3 \sin t
\end{array}\right) \\
& f_{4}(t)=\frac{27 \cdot 10^{-3}}{32}\left(\begin{array}{l}
\left(40 \pi+8-25 t^{2}-(40-50 \pi) t-25 \pi^{2}\right) e^{\pi / 2-t / 2} H(t-\pi) \\
+(8 \cos t-44 \sin t) H(t-\pi)+\left(8-25 t^{2}-40 t\right) e^{-t / 2} \\
-8 \cos t+44 \sin t
\end{array}\right)
\end{aligned}
$$




$$
\left.\begin{array}{rl}
f_{5}(t)=\frac{27 \cdot 10^{-4}}{128}\left(\begin{array}{l}
\left(25\left(t^{3}-\pi^{3}\right)-75(5 \pi-4) t^{2}\right. \\
-5\left(120 \pi+24-75 \pi^{2}\right) t \\
+12\left(25 \pi^{2}+10 \pi-48\right)
\end{array}\right) e^{\pi / 2-t / 2} H(t-\pi) \\
+24(7 \sin t-24 \cos t) H(t-\pi)+\left(125 t^{3}+300 t^{2}-120 t-576\right) e^{-t / 2} \\
+24(24 \cos t-7 \sin t) \\
f_{6}(t)=\frac{81 \cdot 10^{-5}}{2048}\left(\begin{array}{l}
625\left(4 \pi-\frac{16}{5}\right) t^{3}-625 t^{4} \\
+625\left(4 \pi^{3}-\frac{48 \pi^{2}}{5}-\frac{96 \pi}{25}+\frac{2304}{125}\right) t-625 \pi^{4} \\
+625\left(\frac{48 \pi}{5}+\frac{48}{25}-6 \pi^{2}\right) t^{2} \\
+2000 \pi^{3}+1200 \pi^{2}-11520 \pi-7296 \\
+64(114 \cos t+123 \sin t) H(t-\pi) \\
+\left(\begin{array}{l}
1200 t^{2}-625 t^{4}-2000 t^{3} \\
+11520 t+7296
\end{array}\right) e^{-t / 2}-64(114 \cos t-23 \sin t)
\end{array}\right) e^{\pi / 2-t / 2} H(t-\pi)
\end{array}\right)
$$

The M Matrix, Equation (22), and its decomposition, Equation (25), are

$$
\begin{aligned}
\mathbf{M} & =\left[\begin{array}{ccccc}
1.000 & 0 & 0 & 0 & 0 \\
1.000 & 1.000 & 0 & 0 & -0.022801 \\
0 & 1.000 & 1.000 & 0 & -0.187188 \\
0 & 0 & 1.000 & 1.000 & -0.698901 \\
0 & 0 & 0 & 1.000 & -0.305090
\end{array}\right] \\
\mathbf{A} & =\left[\begin{array}{ccccc}
1.000 & 0 & 0 & 0 & 0 \\
0 & 0.776+0.250 i & 0 & 0 & 0 \\
0 & 0 & 0.776-0.250 i & 0 & 0 \\
0 & 0 & 0 & 0.571+0.137 i & 0 \\
0 & 0 & 0 & 0 & 0.571-0.137 i
\end{array}\right]
\end{aligned}
$$




$$
\mathbf{V}=\left[\begin{array}{ccccc}
-0.013 & 0 & 0 & 0 & 0 \\
-0.104 & 0.036+0.025 i & 0.036-0.025 i & 0.035+0.011 i & 0.035-0.011 i \\
-0.389 & 0.277+0.077 i & 0.277-0.077 i & 0.222+0.045 i & 0.222-0.045 i \\
-0.726 & 0.711 & 0.711 & 0.638+0.099 i & 0.638-0.099 i \\
-0.557 & 0.624-0.144 i & 0.624+0.144 i & 0.728 & 0.728
\end{array}\right]
$$

$$
\mathbf{V}^{-1}=\left[\begin{array}{ccccc}
-78.8 & 0 & 0 & 0 & 0 \\
45.2+78.7 i & -29.8-6.33 i & 8.25-6.03 i & -0.34+3.41 i & -0.78-0.85 i \\
45.2-78.7 i & -29.8+6.33 i & 8.25+6.03 i & -0.34-3.41 i & -0.78+0.85 i \\
-84.4+68.9 i & 26.8-41.1 i & -5.87+21.3 i & -0.39-9.92 i & 1.52+4.20 i \\
-84.4-68.9 i & 26.8+41.1 i & -5.87-21.3 i & -0.39+9.92 i & 1.52-4.20 i
\end{array}\right]
$$

